Narrow $\mathrm{Au}(111)$ terraces decorated by self-organized Co nanowires: a low-temperature STM/STS investigation

This article has been downloaded from IOPscience. Please scroll down to see the full text article.

2010 J. Phys.: Condens. Matter 22255504

(http://iopscience.iop.org/0953-8984/22/25/255504)

View the table of contents for this issue, or go to the journal homepage for more

Download details:

IP Address: 134.58.253.57

The article was downloaded on 08/06/2010 at 07:03

Please note that terms and conditions apply. 


\title{
Narrow Au(111) terraces decorated by self-organized Co nanowires: a low-temperature STM/STS investigation
}

\author{
K Schouteden and C Van Haesendonck \\ Laboratory of Solid-State Physics and Magnetism and Institute for Nanoscale Physics and \\ Chemistry (INPAC), BE-3001 Leuven, Belgium \\ E-mail: Koen.Schouteden@fys.kuleuven.be
}

Received 9 March 2010, in final form 9 May 2010

Published 7 June 2010

Online at stacks.iop.org/JPhysCM/22/255504

\begin{abstract}
Deposition of Co atoms on $\mathrm{Au}(111)$ surfaces leads to the formation of self-organized bilayer Co nanowires at the step edges between adjacent narrow $\mathrm{Au}(111)$ terraces. Scanning tunneling microscopy and spectroscopy at low temperatures is used to probe the influence of the finite dimensions on the local density of states for both the Co wires and the narrow Au terraces. Confinement of $\mathrm{Au}$ surface state electrons to narrow Au terraces causes a shift of the Au surface state onset energy to higher energies. For the Co nanowires discrete energy levels are observed. Moreover, standing wave patterns occur at the surface of both the Au and the Co. The patterns increase in complexity with increasing energy. All Co nanowires formed at the edges of narrow $\mathrm{Au}$ terraces reveal a characteristic maximum in the local density of states at a significantly different energy when compared to the Co islands that are formed on large Au terraces. The experimental results can be interpreted in terms of a simple particle-in-a-box model.

S Online supplementary data available from stacks.iop.org/JPhysCM/22/255504/mmedia
\end{abstract}

(Some figures in this article are in colour only in the electronic version)

\section{Introduction}

The growth and characterization of nanoparticles on surfaces has received a continuously increasing interest during the latest decade because of their novel and intriguing electronic and magnetic properties as well as their relevance as future building blocks in nanotechnological applications. Scanning tunneling microscopy (STM) and scanning tunneling spectroscopy (STS) hereby are ideal tools to probe with high spatial and energy resolution the morphology and electronic properties of individual nanoparticles, respectively. Several routes are considered for obtaining nanoparticles of different size and shape. One possible route is to create nanoparticles on surfaces by atom manipulation with an STM tip, yielding atomic size structures that include nanowires [1], corrals [2] and polygons $[3,4]$. Alternatively, nanowires can be obtained by preparing (vicinal) crystalline surfaces with terraces that are only a few atoms wide $[5,6]$.

Here, we have opted for the alternative route of forming nanoparticles by means of self-organization of atoms after deposition on a surface. Among many suited atomically flat (111) oriented surfaces, the $A u(111)$ surface is often considered as an ideal template for the controlled growth of metallic nanoparticles by atom deposition. Deposited atoms self-organize into atomically flat islands that are two monolayers high at the elbows of the well known Au(111) herringbone reconstruction [7]. Various materials have already been shown to yield well organized nanoislands on $\mathrm{Au}(111)$, including Fe [8], Co [9], Ni [10] and Cr [11].

Recently, we have probed by means of STM and STS the electronic [12] and magnetic [13] properties of bilayer Co islands formed on large Au terraces after Co atom deposition. We now demonstrate that for similar Co deposition conditions nanowire-like islands are formed at the step edges between adjacent narrow terraces on the $\mathrm{Au}(111)$ surface. We have performed a detailed investigation of the electronic properties of the narrow $\mathrm{Au}$ terraces and of $\mathrm{Co}$ nanowires at the step edges. Clear effects resulting from confinement of electrons to these nanostructures are observed. Our experimental findings are consistent with the results of a simple particle-in-a-box model. 

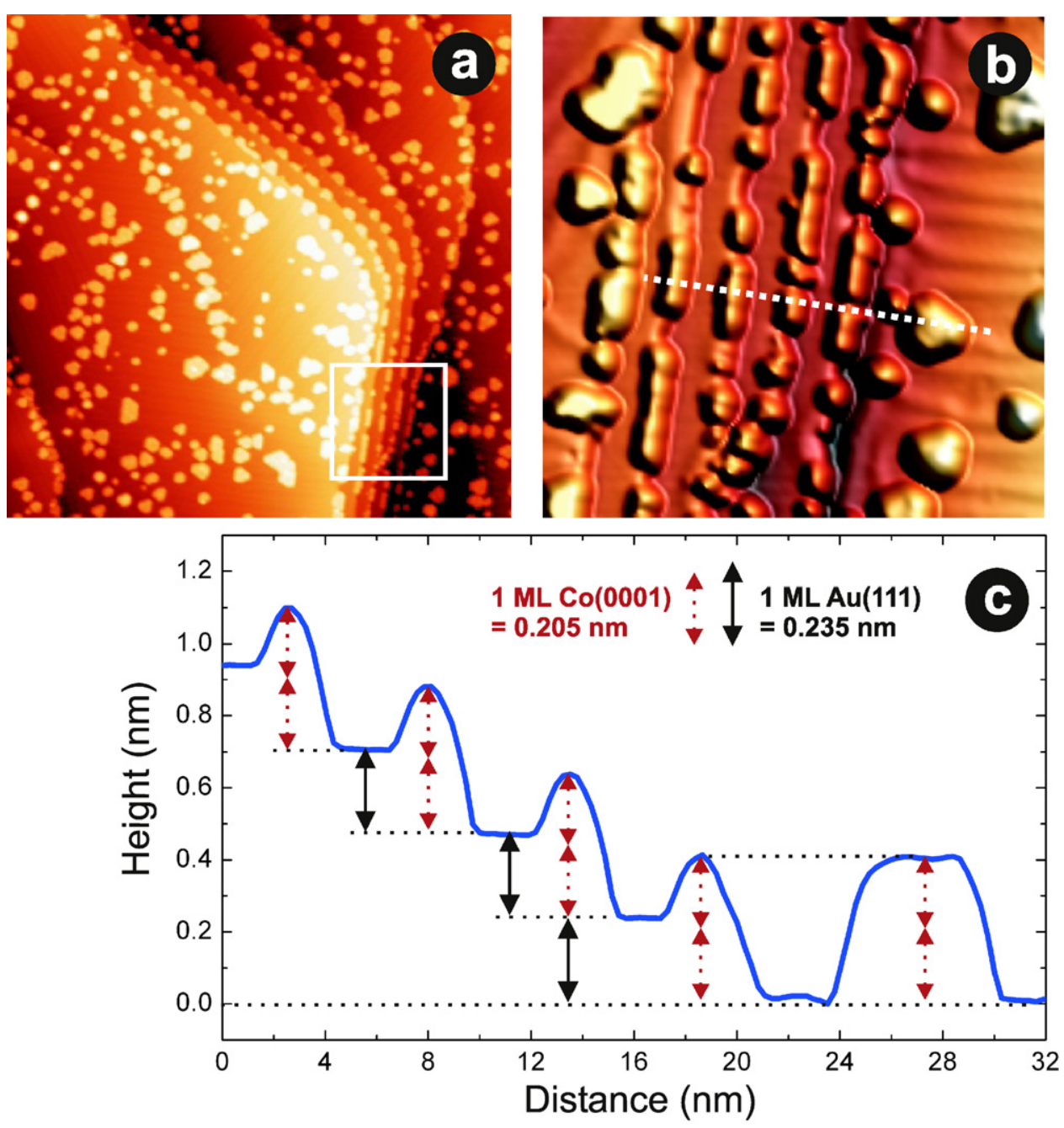

Figure 1. (a) $200 \times 200 \mathrm{~nm}^{2}$ constant current STM image of self-organized Co islands and Co nanowires on Au(111). The Co nanowires are formed at the step edges separating adjacent Au terraces $(I=0.6 \mathrm{nA}, V=+0.8 \mathrm{~V})$. (b) $46 \times 46 \mathrm{~nm}^{2}$ Close-up view of the area marked by the white square in (a). (c) Height profile taken along the white dotted line in (b) $(I=0.6 \mathrm{nA}, V=-0.9 \mathrm{~V})$.

\section{Experimental details}

Atomically flat $\mathrm{Au}(111)$ films are epitaxially grown ex situ on mica and are cleaned in situ by sputtering and annealing cycles, after which Co atoms are deposited at room temperature as described in [12]. The nominal Co coverage is around 0.4 ML $(1 \mathrm{ML}$ of $\mathrm{Co}(0001)=0.205 \mathrm{~nm})$.

STM and STS measurements were performed with a low-temperature STM system (Omicron Nanotechnology), operating under ultra-high vacuum (UHV) conditions at a base pressure in the $10^{-11}$ mbar range. All measurements are performed at $T_{\text {sample }} \simeq 4.5 \mathrm{~K}$. Mechanically cut PtIr (10\% Ir) tips were used in all experiments. Tips were cleaned in situ by applying high voltage pulses up to $10 \mathrm{~V}$ while being in close proximity of a clean $\mathrm{Au}(111)$ surface, until stable topography and spectroscopy can be achieved. The known electron surface state of the clean $\mathrm{Au}(111)$ surface is used as a quality label for our measurements [14]. STM topographic imaging was performed in constant current mode.

Grids of $133 \times 200 \mathrm{~d} I / \mathrm{d} V(V)$ curves are acquired with open feedback loop by means of harmonic detection with a lock-in amplifier at a modulation frequency of $780 \mathrm{~Hz}$ and with a modulation amplitude of $30 \mathrm{mV}$. From these grids, maps of the local density of states (LDOS) are obtained at selected values of the bias voltage $V$. Additionally, LDOS maps are acquired directly with closed feedback loop using tunneling voltage modulation and lock-in amplification based detection at a modulation frequency of $780 \mathrm{~Hz}$ and with a modulation amplitude of $60 \mathrm{mV}$. The bias voltages indicated in the text and figure captions are always with respect to the sample, while the STM tip is virtually grounded. Image processing is performed by Nanotec WSxM [15].

\section{Results}

In figure 1(a) we present a typical STM image of the $\mathrm{Au}(111)$ surface after Co deposition. Deposited Co atoms self-organize into bilayer nanoparticles of only a few nanometers in size at the elbows of the $\mathrm{Au}$ herringbone reconstruction and are referred to as 'Co islands' hereafter. Additionally, Co nanoparticles are formed at the step edges between adjacent, sufficiently narrow $\mathrm{Au}(111)$ terraces. This is illustrated in 
more detail in figure 1(b), which is a zoom-in image of the area enclosed by the white square in figure 1(a). Whereas Co islands exhibit a polygonal shape, the nanoparticles formed at the step edges are clearly elongated along the step edge and exhibit a wire-like shape. Such elongated Co nanoparticles are referred to as 'Co nanowires' hereafter. The nanowires appear to grow more straight when compared to the case of vicinal $\mathrm{Au}(111)$ surfaces [16]. On vicinal $\mathrm{Au}(111)$ surfaces Co islands start to grow upon moderate Co atom deposition at step edges at equidistant locations that are determined by the regular discommensuration lines, which run perpendicular to the vicinal step edges. Upon further Co atom deposition, islands start to interconnect, thereby forming nanowires that consequently have somewhat irregular shapes [16]. For our samples the discommensuration lines (which form the well known herringbone reconstruction on large non-vicinal $\mathrm{Au}(111)$ terraces) appear to be absent on the narrowest $\mathrm{Au}(111)$ terraces in figures 1(a) and (b). This allows the Co atoms to form more elongated nanowires along the $\mathrm{Au}(111)$ step edges. By optimizing our deposition parameters it may be possible to promote the formation of even longer nanowires, but this is beyond the scope of our present work.

From figure 1(b) it may seem at first sight that the Co nanowires are formed at the upper part of the step edges and have a height of only one monolayer. Upon careful inspection of STM height profiles, however, it becomes clear that the Co nanowires have a height of two Co monolayers exclusively, similar to the Co islands [9]. This is illustrated by the height profile in figure 1(c) (taken along the white dotted line in figure 1(b)), which also comprises a bilayer Co island. The formation of a second monolayer has been predicted by theory for Co aggregates on $\mathrm{Au}(111)$ starting from a size of only 20 atoms [17]. For our deposition parameters the width of the nanowires is similar for all nanowires (around $2.0 \mathrm{~nm}$ for full width at half maximum). This width corresponds to about nine Co atoms, implying that the number of atoms forming a single nanowire is indeed well above 20 atoms.

In figure 2(a) (corresponding to the central area in figure 1(b)) we present a topographic image of a series of parallel Co nanowires formed at the step edges separating narrow $\mathrm{Au}$ terraces. In figures 2(b)-(1) we present the corresponding LDOS maps at the indicated tunneling voltages. Both the outer left and right Au terraces have a width of several tens of nanometers (see figure 1(a)). Below the onset of the $\mathrm{Au}(111)$ surface state at $-460 \mathrm{meV}$ [14] no distinct features can be resolved at the $\mathrm{Au}(111)$ terraces. The Co nanowires have a lower LDOS than the surrounding parts of the $\mathrm{Au}$ terraces (figure 2(b)). Above $-460 \mathrm{meV}$ the outer left and right large $\mathrm{Au}$ terraces as well as the wider regions of the narrower terraces reveal a higher LDOS when compared to the rest of the surface (figure 2(c)). This increase in LDOS is related to the existence of surface states that occur above $-460 \mathrm{meV}$. For the narrower regions of the terraces the increase in the LDOS occurs at significantly higher energies (figure 2(d)). At more elevated energies all Au terraces have a rather similar LDOS (figures 2(e)-(1)) and reveal complex standing wave patterns that are most clearly visible in figures $2(\mathrm{j})-(\mathrm{k})$. These patterns are due to scattering of Au surface state electrons at the Au step edges and at the Co nanowires.

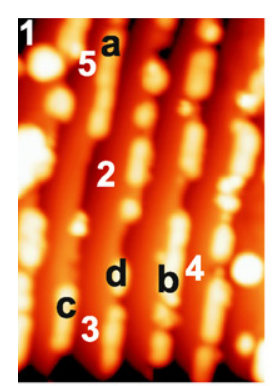

(a)

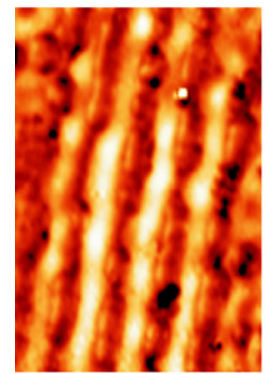

(d)

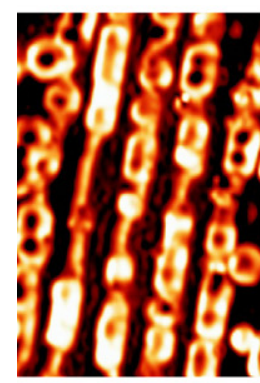

(g)

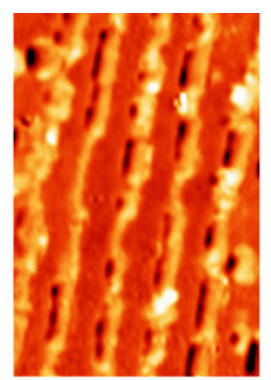

(b)

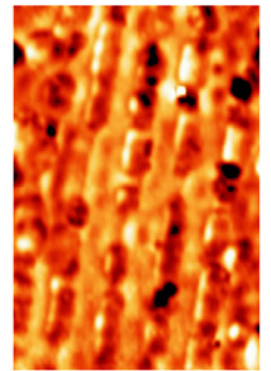

(e)

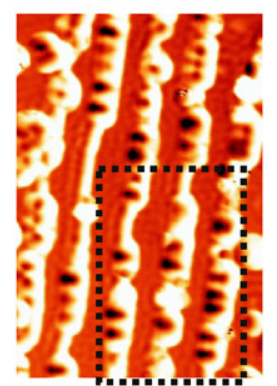

(h)

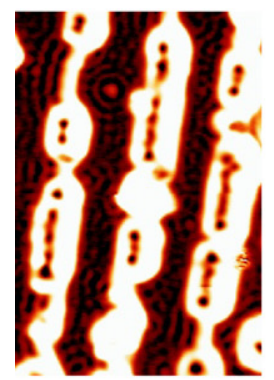

(j)

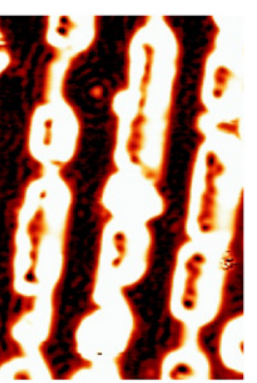

(k)

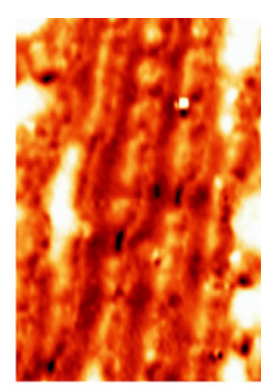

(c)

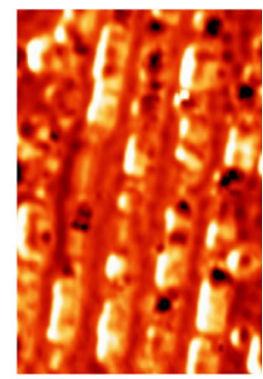

(f)

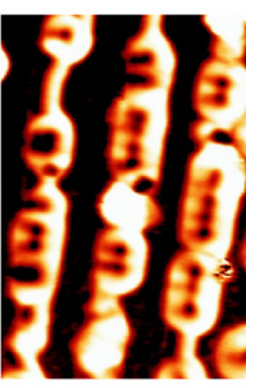

(i)

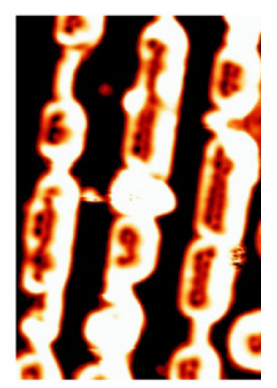

(I)
Figure 2. (a) Constant current STM image of Co nanowires formed at Au step edges and (b)-(l) a corresponding series of LDOS maps at the indicated energies. Images (i)-(l) are recorded at the location marked by the black dotted rectangle in (h). (b)-(g) are obtained from a $(\mathrm{d} I / \mathrm{d} V)(V)$ grid measurement (open feedback loop, $I=0.6 \mathrm{nA}, V=-0.9 \mathrm{~V})$, while (h)-(l) are measured directly (closed feedback loop, $I=3.0 \mathrm{nA}$ ). Image sizes are (a)-(h) $27 \times 40 \mathrm{~nm}^{2}$ and (i)-(l) $16 \times 24 \mathrm{~nm}^{2}$. The LDOS movie is available at stacks.iop.org/JPhysCM/22/255504/mmedia (image size is $27 \times 40 \mathrm{~nm}^{2}$, voltage range from $-0.9 \mathrm{~V}$ to $+1.0 \mathrm{~V}, I=0.6 \mathrm{nA}$, $V=-0.9 \mathrm{~V}$ ). (a) Topography; (b) $-700 \mathrm{meV}$; (c) $-400 \mathrm{meV}$; (d) $-290 \mathrm{meV}$; (e) $-100 \mathrm{meV}$; (f) $+100 \mathrm{meV}$; (g) $+500 \mathrm{meV}$; (h) $+100 \mathrm{meV}$; (i) $+1300 \mathrm{meV}$; (j) $+1800 \mathrm{meV}$; (k) $+2100 \mathrm{meV}$; (l) $+2700 \mathrm{meV}$.

Starting from around $+500 \mathrm{meV}$ electronic features can be resolved within the interior of the Co nanowires as well. Local minima appear along the length of the nanowires as circularly 


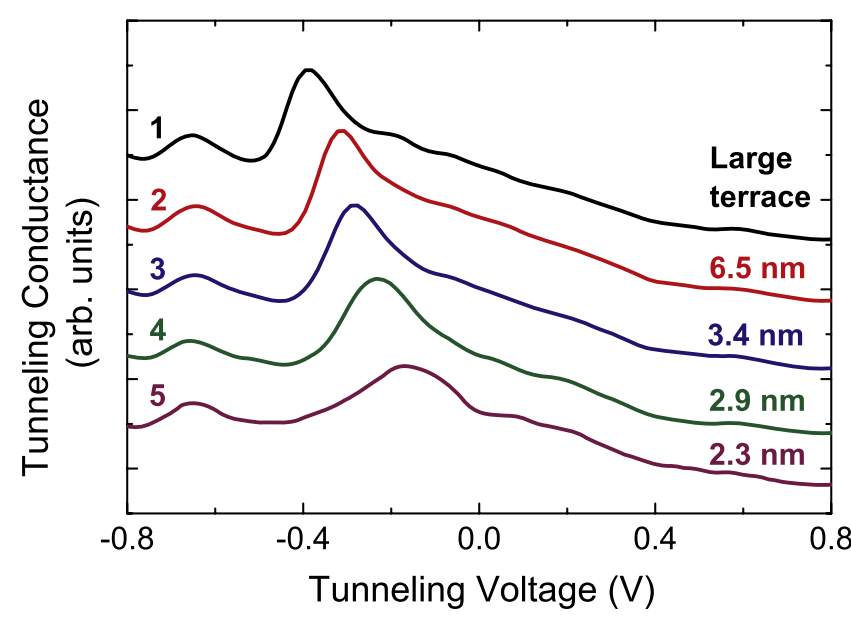

Figure 3. $(\mathrm{d} I / \mathrm{d} V)(V)$ spectra recorded at the correspondingly labeled locations in figure 2(a). The broad maximum is related to the onset of the Au surface state band. Confinement of Au surface state electrons to the narrow Au terraces causes a shift of the maximum to higher energies with decreasing terrace width $(I=0.6 \mathrm{nA}$, $V=-0.9 \mathrm{~V})$.

shaped depressions in the LDOS maps (figures 2(g)-(1)). The number of observed minima strongly depends on the applied tunneling voltage as well as on the length of the nanowire, while the minima have a similar size for all nanowires. These patterns can be attributed to the existence of Co surface state electrons at the Co nanowire surface [12]: interference of forward and backward scattered electrons at both ends of the nanowire results in the formation of standing waves that can be probed by STM. With increasing energy the wavelength of the Co surface state electrons decreases and more complex patterns appear, i.e. the electronic features (the minima) become smaller in size until even multiple minima can be observed along the width of the nanowire (figure 2(1)). For sufficiently low energy values, the wavelength of the electrons is too large when compared to the width of the nanowire to allow formation of standing waves and consequently no patterns can be resolved along the width of the wires.

In figure 3 we present five $(\mathrm{d} I / \mathrm{d} V)(V)$ spectra recorded with open feedback loop at various Au terraces of different width at the correspondingly labeled locations in figure 2(a). The width of the corresponding terrace is indicated for each spectrum. Widths are in the $2-7 \mathrm{~nm}$ range. These widths exceed the characteristic value $\lambda_{\mathrm{F}} / 2$ for which depopulation of the occupied surface state region is predicted [18], where $\lambda_{\mathrm{F}} \simeq$ $3.8 \mathrm{~nm}$ is the Fermi wavelength of Au surface state electrons for large terrace width [12, 19]. Surface state depopulation has recently been observed for the case of Ag surface state electrons confined to very narrow $\mathrm{Ag}(111)$ terraces [5].

Curve (1) in figure 3 has been acquired at a large atomically flat terrace and reveals an onset of the Au surface state around $-440 \mathrm{meV}$ (corresponding to the middle of the step-like rise of the conductivity) with the maximum located around $-390 \mathrm{meV}$. Although the differences in width are rather small, the onset of the surface state is observed to shift to higher energies (up to $-175 \mathrm{meV}$ ) with decreasing terrace width. This behavior can be attributed to confinement of $\mathrm{Au}$ surface state electrons to the terraces having a very small width. A similar behavior has been reported by Morgenstern et al for the case of narrow $\operatorname{Ag}(111)$ terraces [5]. Previously, we reported on similar observations for the case of Au nanowirelike islands [20]. Furthermore, there occurs a small peak around $-650 \mathrm{meV}$ for the $(\mathrm{d} I / \mathrm{d} V)(V)$ spectra in figure 3 . This peak does not appear to be influenced by the onedimensional (1D) confinement imposed by the Au step edges and, since it does not occur for other STM tips, can be attributed to the presence of an electronic state localized at the apex of the STM tip.

Our findings to a certain extent differ from the room temperature measurements reported by Avouris et al for a $3.6 \mathrm{~nm}$ wide Au terrace that did not reveal a shift of the surface state maximum to higher energies, although the electronic features were discussed in terms of a particle-in-a-box-type electron confinement [21]. Data for other terrace widths were, however, not presented by Avouris et al. Thermal drift of the piezo scanner and broadening of the electronic features due to measuring at room temperature $\left(k_{\mathrm{B}} T \simeq 26 \mathrm{meV}\right)$ may account for the different behavior at room temperature.

To the simplest level of approximation, we can model the confinement of the quasi-two-dimensional free-electron-like gas of Au surface state electrons by the edges of the Au terraces in terms of a 1D square well [22]. The nanoscale width $W$ of the well implies a discrete character of its electronic states. Basic quantum mechanics predicts that discrete electronic levels occur at specific energies

$$
E_{n}=E_{0}+\frac{n^{2} \pi^{2} \hbar^{2}}{2 m_{\mathrm{Au}}^{*} W^{2}} \quad n=1,2,3, \ldots,
$$

with $m^{*}$ the effective electron mass and $E_{0}$ the onset energy of the Au surface state. We may approximate $m^{*}$ by its value obtained before for large defect free atomically flat terraces, i.e. $m_{\mathrm{Au}}^{*}=0.23 m_{\mathrm{e}}\left(m_{\mathrm{e}}\right.$ is the free electron mass $)$ [23]. The $\mathrm{Au}$ surface state is known to have an onset energy $E_{0}=$ $-460 \mathrm{meV}$ [14].

In terms of the 1D particle-in-a-box model, the broadened step in the $(\mathrm{d} I / \mathrm{d} V)(V)$ spectra in figure 3 can be interpreted as the first eigenstate of a 1D square well having a width $L$ equal to the width of the Au terraces. According to equation (1), 1D confinement causes an energy shift ranging between 40 and $180 \mathrm{meV}$ with respect to $E_{0}$ for the range of terrace widths that can be observed in figure 2(a). This is in good agreement with the observed shifts of the broadened steps appearing in figure 3 .

In figure 4 (bottom panel) we present $\mathrm{d} I / \mathrm{d} V(V)$ spectra for four different Co nanowires, recorded with open feedback loop at the correspondingly labeled locations in figure 2(a), with the nanowire length indicated for each spectrum. The error for the measured length is around $0.2 \mathrm{~nm}$. The Co nanowires again reveal the tip related peak around $-650 \mathrm{meV}$ that was observed for the Au terraces (see figure 3). On the other hand, another peak around $-320 \mathrm{meV}$ is observed for all nanowires. Previously, it was demonstrated that bilayer Co islands, located at the elbows of the herringbone reconstructed $\mathrm{Au}(111)$ surface, exhibit such a peak at a significantly different energy around $-160 \mathrm{meV}[12,24]$. A curve recorded with the same tip at such a Co island, revealing this peak around 


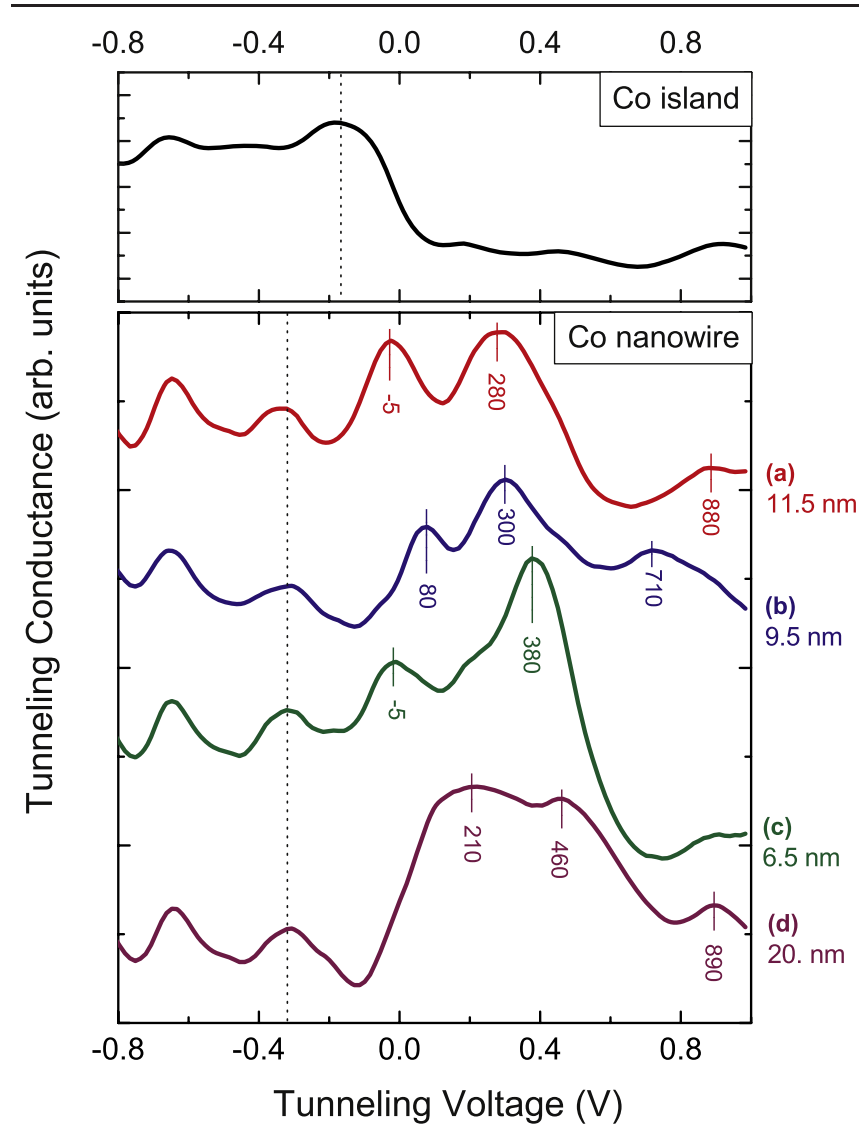

Figure 4. Top panel: $(\mathrm{d} I / \mathrm{d} V)(V)$ spectrum recorded at the center of a Co island that is located at an elbow of the $\mathrm{Au}(111)$ herringbone reconstruction. The black dotted line indicates the position of the surface state related peak around $-160 \mathrm{meV}$. Bottom panel: $(\mathrm{d} I / \mathrm{d} V)(V)$ spectra of Co nanowires formed at Au step edges, recorded at the correspondingly labeled locations in figure 2 (a). The black dotted line indicates the shifted position of the surface state related peak around $-310 \mathrm{meV}(I=0.6 \mathrm{nA}, V=-0.9 \mathrm{~V})$. The energies of the peaks attributed to electron confinement are indicated in meV.

$-160 \mathrm{meV}$, is added as a reference in figure 4 (top panel). A similar peak around $-310 \mathrm{meV}$ was found by Diekhöner et al for bilayer $\mathrm{Co}$ islands on $\mathrm{Cu}(111)$ surfaces [25]. Rastei et al [24] attributed the shift in peak position to a different stacking of the islands on $\mathrm{Au}(111)$ and $\mathrm{Cu}(111)$ surfaces because of the different lattice mismatch. Here, the atomic stacking for large and narrow Au terraces should be similar [7] and therefore cannot account for the change in electronic properties of the Co nanowires when compared to the Co islands. The different electronic properties should therefore rather be related to the modified electronic properties of the narrow underlying $\mathrm{Au}$ terraces when compared to wide Au terraces (see discussion of figure 3 above).

Additional maxima appear at higher energies in the $\mathrm{d} I / \mathrm{d} V(V)$ spectra in figure 4 (bottom panel). The energy of the maxima depends on the length of the nanowire and on the probed location on the surface of the nanowire. We attribute the additional maxima to confinement of Co surface state electrons to the nanosized Co structure, giving rise to discrete energy levels [12].
Although the Co nanowires have an elongated shape, their length must still be considered as finite compared to their width. The vertically confined 2D Co surface state electrons therefore experience additional confinement in both lateral directions of the nanowires. Interpretation of the electronic properties of Co nanowires is hence more appropriate in terms of the 2D variant of the 1D square well potential (equation (1)) that was used for the narrow Au terraces. For a 2D square well potential of width $W$ and length $L$, discrete electron levels are expected at

$$
E_{n m}=E_{0}+\frac{\pi^{2} \hbar^{2}}{2 m_{\mathrm{Co}}^{*}}\left(\frac{n^{2}}{L^{2}}+\frac{m^{2}}{W^{2}}\right) \quad n, m=1,2,3, \ldots,
$$

where the quantum numbers $n$ and $m$ identify the different eigenstates of the rectangular box. Note that for boxes with a very large length to width ratio, equation (2) reduces to equation (1). Obviously there exist no electron states below the onset energy $E_{0}$ of the surface state. It is reasonable to relate this onset energy $E_{0}$ for the Co nanowires to the LDOS maximum that is observed around $-320 \mathrm{meV}$ in the $(\mathrm{d} I / \mathrm{d} V)(V)$ spectra of the nanowires [12]. In this view, the patterns that can be observed at the Co nanowire surface above this onset energy (figures 2(g)-(1)) correspond to the (square of the) electron wavefunctions in the 2D square well potential. The maxima and minima that appear in the LDOS maps along the length of the nanowires then correspond to the maxima/minima and the nodes of the wavefunctions, respectively. By increasing the energy above $E_{0}$, electron levels with increasing quantum numbers $n$ and $m$ are addressed. Taking $E_{0}=-320 \mathrm{meV}, W=2.0 \mathrm{~nm}$, $L=2.0-11.5 \mathrm{~nm}$ and $m_{\mathrm{Co}}^{*}=0.32 m_{\mathrm{e}}$ [12], the first electron state ( $n=1$ and $m=1)$ should be located near the Fermi level. Above this energy, longitudinal LDOS modulations due to electron confinement along the length of the Co nanowires are observed in the LDOS maps in figures $2(\mathrm{~g})-(\mathrm{l})$, corresponding to electron states with $n>1$ and $m=1$. At more elevated energies, electron states with $m=2$ will appear, which are observed as LDOS modulations along the width of the Co nanowires (figures 2(j)-(1)). Our experimental observations qualitatively agree with the 2D square well model, i.e. for the here investigated Co nanowires the calculated energy ranges for the energy levels $E_{11}=0.3-0.8 \mathrm{eV}, E_{21}=$ $0.3-1.5 \mathrm{eV}, E_{12}=1.2-1.5 \mathrm{eV} \cdots$ are consistent with the results presented in figures 2 and 4 . It is not possible to quantitatively link the position of maxima in the bottom panel of figure 4 to eigenstates of the Co nanowires by relying on equation (2) for a rectangular wire because (i) the real nanowire geometry considerably deviates from a perfect rectangle, and (ii) hybridization effects need to be taken into account because of the presence of the d electrons near the Fermi level in Co.

\section{Conclusions}

We have performed a detailed investigation of the electronic properties of narrow $\mathrm{Au}$ terraces and of $\mathrm{Co}$ nanowirelike structures using scanning tunneling microscopy and spectroscopy. Confinement of $\mathrm{Au}(111)$ surface state electrons 
to the $\mathrm{Au}$ terraces having a width of only a few nanometers results in a shift of the onset of the Au surface state towards higher energies. The Co surface state related maximum is observed to shift from $-160 \mathrm{meV}$ for Co islands at large $\mathrm{Au}$ terraces to around $-320 \mathrm{meV}$ for Co nanowires at the step edges between adjacent narrow $\mathrm{Au}$ terraces. Confinement of Co surface state electrons to Co nanowires results in the emergence of discrete energy levels of the nanowires. The electronic properties of both the narrow Au terraces and the Co nanowire-like structures can be interpreted using a simple particle-in-a-box model.

\section{Acknowledgments}

This work has been supported by the Research FoundationFlanders (FWO-Vlaanderen) and by the Belgian Interuniversity Attraction Poles (IAP) and the Flemish Concerted Action (GOA) research programs. KS is a postdoctoral researcher of the FWO.

\section{References}

[1] Nilius N, Wallis T M and Ho W 2002 Science 2971853

[2] Crommie M F, Lutz C P and Eigler D M 1993 Science 262218

[3] Kliewer J, Berndt R and Crampin S 2001 New J. Phys. 322.13

[4] Braun K-F and Rieder K-H 2002 Phys. Rev. Lett. 88096801

[5] Morgenstern K, Braun K-F and Rieder K-H 2002 Phys. Rev. Lett. 89226801

[6] Ortega J E, Ruiz-Osés M, Cordón J, Mugarza A, Kuntze J and Schiller F 2005 New. J. Phys. 7101

[7] Barth J V, Brune H, Ertl G and Behm R J 1990 Phys. Rev. B 429307
[8] Stroscio J A, Pierce D T, Dragoset R A and First P N 1992 J. Vac. Sci. Technol. A 101981

[9] Voigtländer B, Meyer G and Amer N M 1991 Phys. Rev. B 4410354

[10] Chambliss D D, Wilson R J and Chang S 1991 Phys. Rev. Lett. 661721

[11] Schouteden K, Muzychenko D A, Lievens P and Van Haesendonck C 2009 J. Nanosci. Nanotechnol. 96767

[12] Schouteden K, Lijnen E, Janssens E, Ceulemans A, Chibotaru L F, Lievens P and Van Haesendonck C 2008 New J. Phys. 10043016

[13] Schouteden K, Muzychenko D A and Van Haesendonck C 2008 J. Nanosci. Nanotechnol. 83616

[14] Chen W, Madhavan V, Jamneala T and Crommie M F 1998 Phys. Rev. Lett. 801469

[15] Horcas I, Fernandez R, Gomez-Rodriguez J M, Colchero J, Gomez-Herrero J and Baro A M 2007 Rev. Sci. Instrum. 78013705

[16] Repain V, Berroir J, Rousset S and Lecoeur J 2000 Surf. Sci. 447 L152

[17] Chado I, Goyhenex C, Bulou H and Bucher J 2004 Appl. Surf. Sci. 226178

[18] Memmel N and Bertel E 1995 Phys. Rev. Lett. 75485

[19] Petersen L, Laitenberger P, Lægsgaard E and Besenbacher F 1998 Phys. Rev. B 587361

[20] Schouteden K, Lijnen E, Muzychenko D A, Chibotaru L F, Lievens P and Van Haesendonck C 2009 Nanotechnology 20395401

[21] Avouris P and Lyo I W 1994 Science 264942

[22] Griffiths D J 1995 Introduction to Quantum Mechanics (Upper Saddle River, NJ: Prentice-Hall)

[23] Schouteden K, Lievens P and Van Haesendonck C 2009 Phys. Rev. B 79195409

[24] Rastei M V, Bucher J P, Ignatiev P A, Stepanyuk V S and Bruno P 2007 Phys. Rev. B 75045436

[25] Diekhöner L, Schneider M A, Baranow A N, Stepanyuk V S, Bruno P and Kern K 2003 Phys. Rev. Lett. 90236801 